\title{
An individual journey within platial environments of flows
}

\author{
Aleksandra Stanczak ${ }^{\mathrm{a}, *}$, Antoni Moore ${ }^{\mathrm{b}}$ \\ a Artist, LiR3-UMR7366, University of Burgundy, Dijon, France, stanczakalexandra@yahoo.fr \\ ${ }^{b}$ School of Surveying, University of Otago, Dunedin, New Zealand, tony.moore@otago.ac.nz. \\ * Corresponding author
}

Keywords: time-space models, temporal cartography, art \& cartography, climate change

\begin{abstract}
:
The planned 3D exhibition "Journey as a flow" was conceived as a personal self-projection inside of the world of different space-time flows (Stanczak, Moore 2019). The perception of a journey as an emotional and individual experience is at the heart of the phenomenological approach and leads to depiction of dynamic space-time and thematic aspects of our lives. In developing this aspect, we would like to focus below, through artworks, on the transition from the undifferentiated environment to the individualized platial environment of flows endowed with values.
\end{abstract}

Place is a special kind of object. It is a concretion of value, though not a valued thing that can be handled or carried about easily; it is an object in which one can dwell. (Yi-Fu Tuan, 2001)

By following the definition above, we consider all phenomena that happen within place, such as light, colour, architecture, landscape, home, travel, as well as relationship, friendship, power, economy, and so forth as the components of the phenomenological environment because human beings can experience them (Seamon, 2000). From this perspective, movement is the experience of time within place while its character (speed, rhythm, frequency, ....) can be perceived as a flow (Figure.1, Figure.2).

Adding these factors to the time geographic model (Hagerstrand, 1970) for further understanding of how people organize and attach to their environments, in Figure 1 we imagine the potential flows as a cloud. In reality these atmospheric phenomena are made mostly of ice crystals, and when pushed by wind currents, they spread into strands. Their shape is flexible and they have never the same boundaries, however their volume is dictated by winds and downdrafts, just like the movement within environment is dictated by transportation system, available pathways, even weather as well. As a mental symbol they can imitate the platial environment of flows that are potentially opened to changes and are built upon the exact locations in geographic space. Some of travellers can imagine flows more like movements from point A to B and independent to previous and next action, while the others would focus on choices and decisions that accompany these movements as the cause-effect timeline (Figure 2). The individual character of exploration makes the whole process determined by attachment to the environment, and thus, the space-time model that we can associate to it, becomes more and more individual (also Figure 2)..
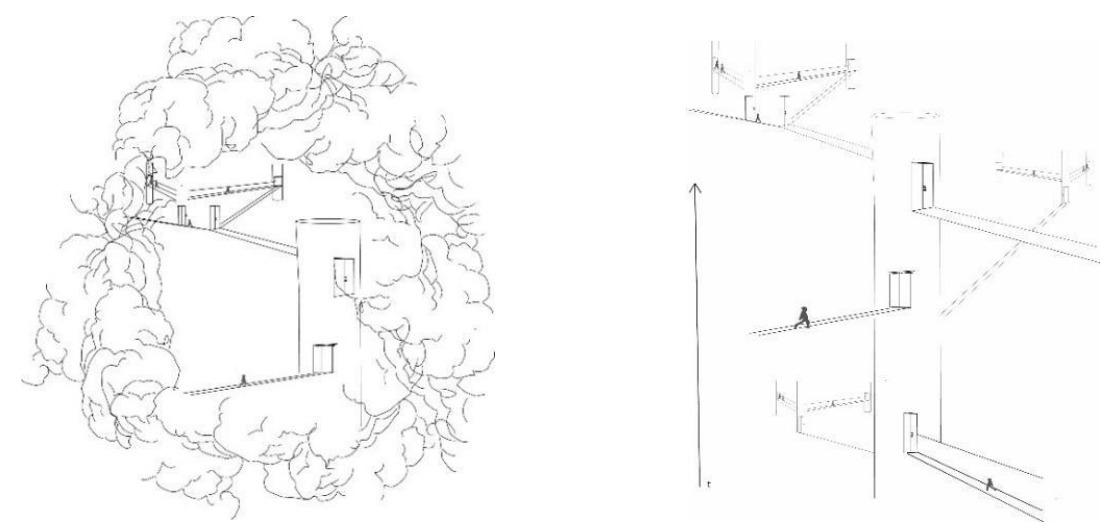

Figure 1. Process of exploration of environment of flows

Figure 2. Model with explicit paths, staircases, stick figures indicating more individualized "time-place". 
Our model of space-time takes into consideration the experience built on the top of the horizontally placed map, like in the time geographic model proposed by Hagerstrand. Also found in the time-geographic model are bundles, indicating possible collaborations of individuals or resources. Figure 3 a presents multiple journeys as separated individual spacetime paths in their own separated world. While they are not subject to the same time-space, as shown in Figure $3 \mathrm{~b}$, which is the evolution of Figure 3a, the configuration of flows within individual environments is different for each of them, even if the bundle is relying different locations into one.

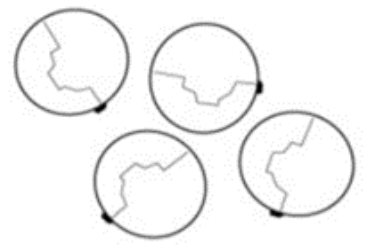

Figure 3a. Multiple journeys in clouds or multiple travelers
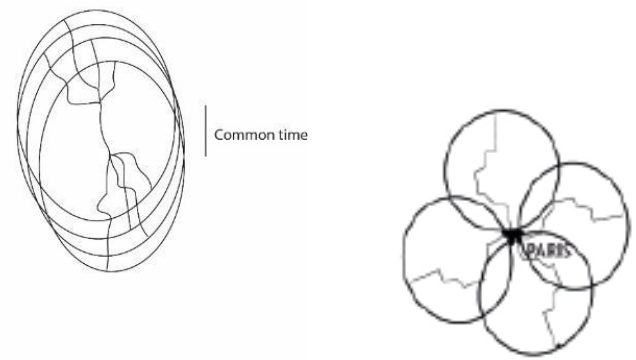

Figure $3 b$ Bundle

From an anthropological point of view the attachment to the environment comes with bonds between individuals (or social groups) and places, as shown in Figure 3b. Thus people can be seen to be dependent upon the concept of place for their self-identity (and social-identity), just as places are dependent upon people for their identity. (Relph, 1976). Relph clearly distinguishes the "location" from the concept of "place" by explaining that a location is where certain specific interactions occur. We would argue then, that bundles are potentially transforming a location into a platial environment and thus they influence on emotional attachment to the specific locations.

Furthermore, by focusing not on distances but more on configurations of flows, we might discover existence of "bridges" between distant places. As shown in Figure 4, in context of Australia one city can be seen as potentially connecting others, because of existence of individual flows.

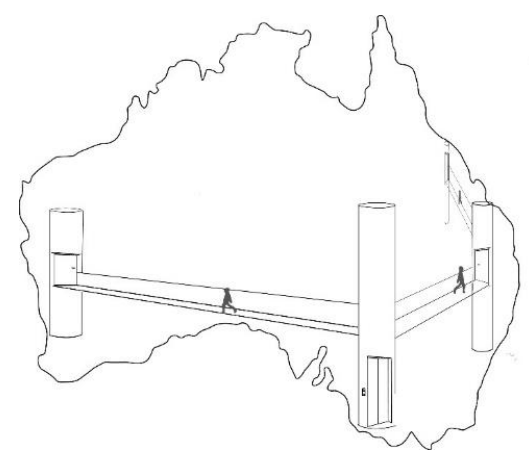

Figure 4. Flows as "bridges" between distant places in context of Australia

We assume that various configurations of individual flows within environments are often playing a crucial role in determining whether the societies are able to transfer their activities in other locations. (e.g. Oakes 2019, Tabucanon 2012) For better cultural understanding of origins of individual potential vulnerability to migrations induced by climate change we propose to discuss the "Journey as a flow" exhibition proposal around these subjects, as well. Adding artistic expression allows us to focus on the relationship between the individual and his living space, and on creating models of perceived and mental realities. We hope to provide a useful conceptual tool and reveal subjective understandings of one position on climate change and mobility to facilitate the planning and implementation of effective policy. 